\title{
SINGULAR PROBLEMS MODELLING PHENOMENA IN THE THEORY OF PSEUDOPLASTIC FLUIDS
}

\author{
RAVI P. AGARWAL ${ }^{1}$ and DONAL O'REGAN ${ }^{2}$
}

(Received 19 October, 2001; revised 14 May, 2002)

\begin{abstract}
Existence criteria are presented for nonlinear singular initial and boundary value problems. In particular our theory includes a problem arising in the theory of pseudoplastic fluids.
\end{abstract}

\section{Introduction}

This paper is motivated by the boundary value problem

$$
\left\{\begin{array}{l}
y^{1 / n} y^{\prime \prime}+n t=0, \quad 0<t<1 \\
y^{\prime}(0)=y(1)=0
\end{array}\right.
$$

which arises in the theory of pseudoplastic fluids. In particular we present existence theory for the mixed boundary value problem

$$
\left\{\begin{array}{l}
\frac{1}{p}\left(p y^{\prime}\right)^{\prime}+q(t) f(t, y)=0, \quad 0<t<1 \\
\lim _{t \rightarrow 0^{+}} p(t) y^{\prime}(t)=y(1)=0
\end{array}\right.
$$

where $f:[0,1] \times(0, \infty) \rightarrow \mathbf{R}$ is continuous. Notice $f$ may be singular at $y=0$. Problems of the above form have been discussed extensively in the literature (see [2-11]) usually when $f$ is positone, that is, $f:(0,1) \times(0, \infty) \rightarrow(0, \infty)$. Only a handful of papers (see [3-5] and the references therein) have appeared where the nonlinearity $f$ is allowed to change sign. This paper presents a new theory, with the idea being to approximate the singular problem by a sequence of nonsingular

\footnotetext{
'Department of Mathematical Sciences, Florida Institute of Technology, Melbourne, FL 32901-6975, USA; e-mail: agarwal@fit.edu.

${ }^{2}$ Department of Mathematics, National University of Ireland, Galway, Ireland; e-mail: donal.oregan@nuigalway.ie.

(C) Australian Mathematical Society 2003, Serial-fee code 1446-1811/03
} 
problems each of which has a lower solution $\alpha_{m}$ and a upper solution $\beta$, and then use a limiting argument. This seems to be more natural and more general than the theory presented in [3-5] since the study of lower solutions to nonsingular problems is well documented. Also in this paper we discuss the singular initial value problem

$$
\left\{\begin{array}{l}
y^{\prime}=q(t) f(t, y), \quad 0<t<T(<\infty) \\
y(0)=0 .
\end{array}\right.
$$

For the remainder of this section we describe the physical problem which motivates our study. The boundary layer equations for steady flow over a semi-infinite plate [1] are

$$
\begin{aligned}
U \frac{\partial U}{\partial X}+V \frac{\partial U}{\partial Y} & =\frac{1}{\rho} \frac{\partial \tau_{X Y}}{\partial Y} \\
\frac{\partial U}{\partial X}+\frac{\partial V}{\partial Y} & =0
\end{aligned}
$$

where the $X$ and $Y$ axes are taken along and perpendicular to the plate, $\rho$ is the density, $U$ and $V$ are the velocity components parallel and normal to the plate and the shear stress $\tau_{X Y}=K(\partial U / \partial Y)^{n}$. The case $n=1$ corresponds to a Newtonian fluid and for $0<n<1$ the power law relation between shear stress and rate of strain describes pseudoplastic non-Newtonian fluids. The fluid has zero velocity on the plate and the flow approaches stream conditions far from the plate, that is,

$$
U(X, 0)=V(X, 0)=0, \quad U(X, \infty)=U_{\infty}
$$

where $U_{\infty}$ is the uniform potential flow. The above results (if we use stream functionsimilarity variables) $[1,9]$ in a third-order infinite interval problem

$$
F^{\prime \prime \prime}+F\left(F^{\prime \prime}\right)^{2-n}=0, \quad F(0)=F^{\prime}(0)=0, \quad F^{\prime}(\infty)=1 .
$$

Now use the Crocco-type transformation $u=F^{\prime}$ and $G=F^{\prime \prime}$ to obtain

$$
G^{n} G^{\prime \prime}+(n-1) G^{n-1}\left(G^{\prime}\right)^{2}+u=0, \quad G^{\prime}(0)=0, \quad G(1)=0
$$

Setting $y=G^{n}$ we obtain

$$
\left\{\begin{array}{l}
y^{1 / n} y^{\prime \prime}+n u=0, \quad 0<u<1 \\
y^{\prime}(0)=y(1)=0 .
\end{array}\right.
$$




\section{Mixed boundary value problems}

Motivated by the example in Section 1 concerning non-Newtonian fluids, we consider the mixed boundary value problem

$$
\left\{\begin{array}{l}
\frac{1}{p}\left(p y^{\prime}\right)^{\prime}+q(t) f(t, y)=0, \quad 0<t<1 \\
\lim _{t \rightarrow 0^{+}} p(t) y^{\prime}(t)=y(1)=0 .
\end{array}\right.
$$

We note also that we do not assume $\int_{0}^{1} d s / p(s)<\infty$. For our first result in this section we will assume the following conditions are satisfied:

$$
\begin{gathered}
p \in C[0,1] \cap C^{1}(0,1) \quad \text { with } p>0 \text { on }(0,1) \\
q \in C(0,1) \quad \text { with } q>0 \text { on }(0,1) \\
\int_{0}^{1} p(s) q(s) d s<\infty \text { and } \int_{0}^{1} \frac{1}{p(t)} \int_{0}^{t} p(s) q(s) d s d t<\infty \\
f:[0,1] \times(0, \infty) \rightarrow \mathbf{R} \text { is continuous }
\end{gathered}
$$

$\left\{\begin{array}{l}\exists n_{0} \in\{1,2, \ldots\} \text { and associated with each } m \in N_{0}=\left\{n_{0}, n_{0}+1, \ldots\right\}, \\ \exists \alpha_{m} \in C[0,1] \cap C^{2}(0,1), p \alpha_{m}^{\prime} \in A C[0,1], \\ \text { with } p(t) q(t) f\left(t, \alpha_{m}(t)\right)+\left(p(t) \alpha_{m}^{\prime}(t)\right)^{\prime} \geq 0 \text { for } t \in(0,1), \\ \lim _{t \rightarrow 0^{+}} p(t) \alpha_{m}^{\prime}(t) \geq 0 \text { and } 0<\alpha_{m}(1) \leq 1 / m\end{array}\right.$

$$
\begin{gathered}
\left\{\begin{array}{l}
\exists \alpha \in C[0,1], \alpha>0 \text { on }[0,1) \text { and } \alpha(t) \leq \alpha_{m}(t), \\
t \in[0,1] \text { for each } m \in N_{0}
\end{array}\right. \\
\left\{\begin{array}{l}
\exists \beta \in C[0,1] \cap C^{2}(0,1), p \beta^{\prime} \in A C[0,1] \text { with } \\
p(t) q(t) f(t, \beta(t))+\left(p(t) \beta^{\prime}(t)\right)^{\prime} \leq 0 \text { for } t \in(0,1), \\
\lim _{t \rightarrow 0^{+}} p(t) \beta^{\prime}(t) \leq 0 \text { and } \beta(1) \geq \beta_{0}>0
\end{array}\right.
\end{gathered}
$$

and

$$
\alpha_{m}(t) \leq \beta(t), \quad t \in[0,1] \text { for each } m \in N_{0}
$$

THEOREM 2.1. (I) Suppose (2.2)-(2.9) hold and in addition assume the following condition is satisfied:

$$
\left\{\begin{array}{l}
0 \leq f(t, y) \leq g(y) \text { on }[0,1] \times\left(0, a_{0}\right] \text { with } g>0 \\
\text { continuous and nonincreasing on }(0, \infty)
\end{array}\right.
$$

here $a_{0}=\sup _{t \in[0.1]} \beta(t)$. Then (2.1) has a solution $y \in C[0,1] \cap C^{2}(0,1)$ with $y(t) \geq \alpha(t)$ for $t \in[0,1]$. 
(II) Suppose (2.2)-(2.9) hold and in addition assume the following condition is satisfied:

$$
f(t, x)-f(t, y)>0 \text { for } 0<x<y \text {, for each fixed } t \in(0,1) .
$$

Then (2.1) has a solution $y \in C[0,1] \cap C^{2}(0,1)$ with $y(t) \geq \alpha(t)$ for $t \in[0,1]$.

PROOF. Without loss of generality assume $\beta_{0} \geq 1 / n_{0}$. Fix $m \in N_{0}$ and consider the boundary value problem

$$
\left\{\begin{array}{l}
\left(p y^{\prime}\right)^{\prime}+p q f_{m}^{\star}(t, y)=0, \quad 0<t<1 \\
\lim _{t \rightarrow 0^{+}} p(t) y^{\prime}(t)=0 \\
y(1)=1 / m
\end{array}\right.
$$

where

$$
f_{m}^{*}(t, y)= \begin{cases}f(t, \beta(t))+r(\beta(t)-y), & y>\beta(t) \\ f(t, y), & \alpha_{m}(t) \leq y \leq \beta(t) \\ f\left(t, \alpha_{m}(t)\right)+r\left(\alpha_{m}(t)-y\right), & y<\alpha_{m}(t)\end{cases}
$$

with $r: \mathbf{R} \rightarrow[-1,1]$ the radial retraction defined by

$$
r(u)= \begin{cases}u, & |u| \leq 1 \\ u /|u|, & |u|>1\end{cases}
$$

It is immediate from Schauder's fixed point theorem (see [10]) that $(2.12)^{m}$ has a solution $y_{m} \in C[0,1]$ (in fact $y_{m} \in C[0,1] \cap C^{2}(0,1)$ with $p y_{m}^{\prime} \in A C[0,1]$ ). A standard argument (see [10, Chapter 5]; note $f_{m}^{\star}:[0,1] \times \mathbf{R} \rightarrow \mathbf{R}$ is continuous) guarantees that

$$
\alpha_{m}(t) \leq y_{m}(t) \leq \beta(t) \quad \text { for } t \in[0,1]
$$

As a result $y_{m}$ is a solution of

$$
\left\{\begin{array}{l}
\left(p y^{\prime}\right)^{\prime}+p q f(t, y)=0, \quad 0<t<1 \\
\lim _{t \rightarrow 0^{+}} p(t) y^{\prime}(t)=0 \\
y(1)=1 / m
\end{array}\right.
$$

In addition (2.7) guarantees that

$$
\alpha(t) \leq \alpha_{m}(t) \leq y_{m}(t) \leq \beta(t) \quad \text { for } t \in[0,1] .
$$

The proof is now broken into two cases. 
Case (A). Suppose (2.10) holds.

We first show

$$
\left\{y_{m}\right\}_{m \in N_{0}} \text { is a bounded, equicontinuous family on }[0,1] \text {. }
$$

First notice from (2.10) that $\left(p y_{m}^{\prime}\right)^{\prime} \leq 0$ on $(0,1)$, so $p y_{m}^{\prime} \leq 0$ on $(0,1)$. In addition $-\left(p(t) y_{m}^{\prime}(t)\right)^{\prime} \leq p(t) q(t) g\left(y_{m}(t)\right)$ for $t \in(0,1)$, so integration from 0 to $t$ yields

$$
-p(t) y_{m}^{\prime}(t) \leq g\left(y_{m}(t)\right) \int_{0}^{t} p(s) q(s) d s \text { for } t \in(0,1) .
$$

As a result

$$
0 \leq \frac{-y_{m}^{\prime}(t)}{g\left(y_{m}(t)\right)} \leq \frac{1}{p(t)} \int_{0}^{t} p(s) q(s) d s \text { for } t \in(0,1)
$$

Now consider $I(z)=\int_{0}^{z} d u / g(u)$. For $t, s \in[0,1]$ we have

so

$$
\left|I\left(y_{m}(t)\right)-I\left(y_{m}(s)\right)\right|=\left|\int_{s}^{t} \frac{y_{m}^{\prime}(x)}{g\left(y_{m}(x)\right)}\right| \leq\left|\int_{s}^{t} \frac{1}{p(x)} \int_{0}^{x} p(z) q(z) d z d x\right|,
$$

$$
\left\{I\left(y_{m}\right)\right\}_{m \in N_{0}} \text { is a bounded, equicontinuous family on }[0,1] \text {. }
$$

The uniform continuity of $I^{-1}$ on $\left[0, I\left(a_{0}\right)\right]$ together with $(2.17)$ and

$$
\left|y_{m}(t)-y_{m}(s)\right|=\left|I^{-1}\left(I\left(y_{m}(t)\right)\right)-I^{-1}\left(I\left(y_{m}(s)\right)\right)\right|
$$

guarantees (2.16). A standard argument [2, page 90] using the Arzela-Ascoli theorem (and (2.15)) completes the proof.

Case (B). Suppose (2.11) holds.

We begin by showing

$$
y_{m+1}(t) \leq y_{m}(t) \text { for } t \in[0,1] \text { for each } m \in N_{0} .
$$

Suppose (2.18) is false. Then for some $m \in N_{0}, y_{m+1}-y_{m}$ would have a positive absolute maximum at say $\tau_{0} \in[0,1)$. Suppose to begin with $\tau_{0} \in(0,1)$, so $\left(y_{m+1}-\right.$ $\left.y_{m}\right)^{\prime}\left(\tau_{0}\right)=0$ and $\left(p\left(y_{m+1}-y_{m}\right)^{\prime}\right)^{\prime}\left(\tau_{0}\right) \leq 0$. On the other hand, (2.11) implies

$$
\left(p\left(y_{m+1}-y_{m}\right)^{\prime}\right)^{\prime}\left(\tau_{0}\right)=-p\left(\tau_{0}\right) q\left(\tau_{0}\right)\left[f\left(\tau_{0}, y_{m+1}\left(\tau_{0}\right)\right)-f\left(\tau_{0}, y_{m}\left(\tau_{0}\right)\right)\right]>0,
$$

a contradiction. If $\tau_{0}=0$ then $\lim _{t \rightarrow 0^{+}} p(t)\left[y_{m+1}-y_{m}\right]^{\prime}(t)=0$ and there exists $\mu>0$ with $y_{m+1}(s)-y_{m}(s)>0$ for $s \in(0, \mu)$. Thus for $t \in(0, \mu)$ we have from $(2.11)$ that

$$
p\left(y_{m+1}-y_{m}\right)^{\prime}(t)=\int_{0}^{t} p(s) q(s)\left[f\left(s, y_{m}(s)\right)-f\left(s, y_{m+1}(s)\right)\right] d s>0,
$$


a contradiction since $y_{m+1}-y_{m}$ has a positive absolute maximum at 0 . As a result (2.18) holds.

Lets look at the interval $\left[0,1-1 / n_{0}\right]$. Let

$$
R_{n_{0}}=\sup \left\{|f(t, y)|: t \in\left[0,1-1 / n_{0}\right] \text { and } \alpha(t) \leq y \leq a_{0}\right\}
$$

here $a_{0}=\sup _{t \in[0,1]} \beta(t)$. In addition

$$
\left|y_{m}^{\prime}(t)\right| \leq \frac{R_{n_{0}}}{p(t)} \int_{0}^{t} p(s) q(s) d s \quad \text { for } t \in\left(0,1-1 / n_{0}\right) .
$$

Thus $\left\{y_{m}\right\}_{m \in N_{0}}$ is a bounded, equicontinuous family on $\left[0,1-1 / n_{0}\right]$. The ArzelaAscoli theorem guarantees the existence of a subsequence $N_{n_{0}}$ of $N_{0}$ and a function $z_{n_{0}} \in C\left[0,1-1 / n_{0}\right]$ with $y_{m}$ converging uniformly on $\left[0,1-1 / n_{0}\right]$ to $z_{n_{0}}$ as $m \rightarrow \infty$ through $N_{n_{0}}$. Proceed inductively to obtain subsequences of integers

$$
N_{n_{0}} \supseteq N_{n_{0}+1} \supseteq \cdots \supseteq N_{k} \supseteq \cdots
$$

and functions $z_{k} \in C[0,1-1 / k]$ with $y_{m}$ converging uniformly on $[0,1-1 / k]$ to $z_{k}$ as $m \rightarrow \infty$ through $N_{k}$, and $z_{k+1}=z_{k}$ on $[0,1-1 / k]$.

Define a function $y:[0,1] \rightarrow[0, \infty)$ by $y(x)=z_{k}(x)$ on $[0,1-1 / k]$ and $y(1)=0$. Notice $y$ is well-defined and $\alpha(t) \leq y(t) \leq a_{0}$ for $t \in[0,1)$. Next fix $t \in(0,1)$ and let $k \in\left\{n_{0}, n_{0}+1, \ldots\right\}$ be such that $0<t<1-1 / k$. Let $N_{k}^{*}=\left\{n \in N_{k}: n \geq k\right\}$. Now $y_{m}, m \in N_{k}^{*}$, satisfies

$$
y_{m}(t)=y_{m}(0)-\int_{0}^{t} \frac{1}{p(s)} \int_{0}^{s} p(x) q(x) f\left(x, y_{m}(x)\right) d x d s .
$$

Let $m \rightarrow \infty$ through $N_{k}^{*}$ to obtain

$$
y(t)=y(0)-\int_{0}^{t} \frac{1}{p(s)} \int_{0}^{s} p(x) q(x) f(x, y(x)) d x d s .
$$

We can do this argument for each $t \in(0,1)$, so $\left(p y^{\prime}\right)^{\prime}(t)+p(t) q(t) f(t, y(t))=0$ for $t \in(0,1)$ and $\lim _{t \rightarrow 0^{+}} p(t) y^{\prime}(t)=0$.

It remains to show $y$ is continuous at 1 . Let $\epsilon>0$ be given. Now since $\lim _{m \rightarrow \infty} y_{m}(1)=0$ there exists $n_{1} \in N_{0}$ with $y_{n_{1}}(1)<\epsilon / 2$. Also since $y_{n_{1}} \in C[0,1]$ there exists $\delta_{n_{1}}>0$ with $y_{n_{1}}(t)<\epsilon / 2$ for $t \in\left[1-\delta_{n_{1}}, 1\right]$. From (2.18) for $m \geq n_{1}$ we have $y_{m}(t) \leq y_{n_{1}}(t)<\epsilon / 2$ for $t \in\left[1-\delta_{n_{1}}, 1\right]$. As a result for $m \geq n_{1}$ we have

$$
0 \leq \alpha(t) \leq y_{m}(t)<\epsilon / 2 \text { for } t \in\left[1-\delta_{n_{1}}, 1\right] \text {. }
$$

Consequently

$$
0 \leq \alpha(t) \leq y(t) \leq \epsilon / 2<\epsilon \text { for } t \in\left[1-\delta_{n_{1}}, 1\right),
$$

so $y$ is continuous at 1 . 
REMARK 2.1. In Theorem 2.1 (I) we can replace (2.10) with

$$
\left\{\begin{array}{l}
|f(t, y)| \leq g(y) \text { on }[0,1] \times\left(0, a_{0}\right] \text { with } g>0 \\
\text { continuous and nonincreasing on }(0, \infty)
\end{array}\right.
$$

and

$$
\int_{0}^{1} \frac{1}{p(s)} \int_{0}^{s} p(x) q(x) g(\alpha(x)) d x d s<\infty
$$

here $a_{0}=\sup _{t \in[0.1]} \beta(t)$. Notice we only used (2.10) to show (2.16). If we assume (2.20) and (2.21) then (2.16) is immediate since

$$
\pm\left(p(t) y_{m}^{\prime}(t)\right)^{\prime} \leq p(t) q(t) g\left(y_{m}(t)\right) \leq p(t) q(t) g(\alpha(t)) \text { for } t \in(0,1),
$$

so

$$
\left|y_{m}^{\prime}(t)\right| \leq \frac{1}{p(t)} \int_{0}^{t} p(s) q(s) g(\alpha(s)) d s \quad \text { for } t \in(0,1) .
$$

We next state and prove a more general result motivated from Theorem 2.1 (II).

THEOREM 2.2. Suppose (2.2)-(2.7) hold and in addition assume the following conditions are satisfied:

$$
\left\{\begin{array}{l}
\text { for each } m \in N_{0}, \exists \beta_{m} \in C[0,1] \cap C^{2}(0,1), p \beta_{m}^{\prime} \in A C[0,1] \\
\text { with } p(t) q(t) f\left(t, \beta_{m}(t)\right)+\left(p(t) \beta_{m}^{\prime}(t)\right)^{\prime} \leq 0 \text { for } t \in(0,1), \\
\lim _{t \rightarrow 0^{+}} p(t) \beta_{m}^{\prime}(t) \leq 0 \text { and } \beta_{m}(1) \geq 1 / m \\
\qquad \alpha_{m}(t) \leq \beta_{m}(t), \quad t \in[0,1] \text { for each } m \in N_{0}
\end{array}\right.
$$

and

$$
\left\{\begin{array}{l}
\text { for each } t \in[0,1] \text { we have that }\left\{\beta_{m}(t)\right\}_{m \in N_{0}} \text { is a } \\
\text { nonincreasing sequence and } \lim _{m \rightarrow \infty} \beta_{m}(1)=0 .
\end{array}\right.
$$

Then (2.1) has a solution $y \in C[0,1] \cap C^{2}(0,1)$ with $y(t) \geq \alpha(t)$ for $t \in[0,1]$.

PROOF. Fix $m \in N_{0}$. Proceed as in Theorem 2.1 with $\beta_{m}$ replacing $\beta$ in $f_{m}^{\star}$. The same reasoning as in Theorem 2.1 guarantees that there exists a solution $y_{m} \in C[0,1]$ to (2.14) with $\alpha(t) \leq \alpha_{m}(t) \leq y_{m}(t) \leq \beta_{m}(t)$ for $t \in[0,1]$. Also as in Theorem 2.1 (from (2.19) onwards) there exists $y \in C[0,1$ ) (as described in Theorem 2.1 (II)) with

$$
\alpha(t) \leq y(t) \leq a_{0}=\sup _{t \in[0,1]} \beta_{n_{0}}(t) \text { for } t \in[0,1),
$$

with $\left(p y^{\prime}\right)^{\prime}(t)+p(t) q(t) f(t, y(t))=0,0<t<1$ and $\lim _{t \rightarrow 0^{+}} p(t) y^{\prime}(t)=0$. 
It remains to show $y$ is continuous at 1 . Let $\epsilon>0$ be given. Now since $\lim _{m \rightarrow \infty} \beta_{m}(1)=0$ there exists $n_{1} \in N_{0}$ with $\beta_{n_{1}}(1)<\epsilon / 2$, and so there exists $\delta_{n_{1}}>0$ with $\beta_{n_{1}}(t)<\epsilon / 2$ for $t \in\left[1-\delta_{n_{1}}, 1\right]$. From (2.24) for $m \geq n_{1}$ we have

$$
\alpha(t) \leq \alpha_{m}(t) \leq y_{m}(t) \leq \beta_{m}(t) \leq \beta_{n_{1}}(t)<\epsilon / 2 \text { for } t \in\left[1-\delta_{n_{1}}, 1\right]
$$

That is, for $m \geq n_{1}$ we have $0 \leq \alpha(t) \leq y_{m}(t)<\epsilon / 2$ for $t \in\left[1-\delta_{n_{1}}, 1\right]$. Consequently $0 \leq \alpha(t) \leq y(t) \leq \epsilon / 2<\epsilon$ for $t \in\left[1-\delta_{n_{1}}, 1\right)$, so $y$ is continuous at 1 .

EXAMPLE (Fluid problem). Consider the boundary value problem

$$
\left\{\begin{array}{l}
y^{\prime \prime}+v t / y^{1 / v}=0, \quad 0<t<1 \\
y^{\prime}(0)=y(1)=0
\end{array}\right.
$$

where $0<v \leq 1$. We will show using Theorem 2.1 (part (I) or (II)) that (2.26) has a solution.

First we choose $n_{0} \in\{1,2, \ldots\}$ so that

$$
\frac{\nu}{6}+\frac{1}{n_{0}} \leq 1 \text { and }\left(\frac{\nu}{6}-1\right) \frac{1}{\nu+1}+\frac{1}{n_{0}} \leq 0 .
$$

Let $p=1, q(t)=2 t$ and clearly (2.2)-(2.5) hold. Also let

$$
\begin{aligned}
\alpha_{m}(t) & =v\left(1-t^{3}\right) / 6+1 / m, \\
\alpha(t) & =v\left(1-t^{3}\right) / 6
\end{aligned}
$$

and $\beta(t)=1-\nu t^{3} /(\nu+1)$. To check (2.6), for $m \in N_{0}=\left\{n_{0}, n_{0}+1, \ldots\right\}$, notice $\alpha_{m}(1)=1 / m, \alpha_{m}^{\prime}(0)=0$ and

$$
\alpha_{m}^{\prime \prime}+q f\left(t, \alpha_{m}\right)=-v t+\frac{v t}{\left[\alpha_{m}(t)\right]^{1 / \nu}} \geq-v t+v t=0 \quad \text { for } t \in(0,1)
$$

since $\alpha_{m}(t) \leq v / 6+1 / n_{0} \leq 1, t \in[0,1]$ from (2.27). Thus (2.6) holds and (2.7) is immediate. To check (2.8) notice $\beta(1)=1-v / \nu+1 \equiv \beta_{0}, \beta^{\prime}(0)=0$ and

$$
\begin{aligned}
\beta^{\prime \prime}+q f(t, \beta) & =\frac{-6 v t}{v+1}+\frac{v t}{[\beta(t)]^{1 / v}} \leq \frac{-6 v t}{v+1}+v t(\nu+1)^{1 / \nu} \\
& =v t\left\{\frac{-6}{v+1}+(\nu+1)^{1 / \nu}\right\} \leq 0 \text { for } t \in(0,1)
\end{aligned}
$$

since $\beta(t) \geq 1 /(v+1)$ for $t \in[0,1]$, and $(v+1)^{(v+1) / v} \leq 4 \leq 6$ for $0<v \leq 1$ (note with $f(x)=(x+1)^{(x+1) / x}$ we have $f\left(0^{+}\right)=e, f(1)=4$ and $f^{\prime}(x) \geq 0$ on $\left.(0,1)\right)$. 
Thus (2.8) holds. In addition (2.9) is true since (2.27) implies for $m \in N_{0}$ that

$$
\begin{aligned}
\alpha_{m}(t) & =\frac{v}{6}\left(1-t^{3}\right)+\frac{1}{m} \leq \frac{v}{6}\left(1-\frac{v}{v+1} t^{3}\right)+\frac{1}{n_{0}} \\
& =\frac{v}{6} \beta(t)+\frac{1}{n_{0}}=\beta(t)+\left\{\frac{1}{n_{0}}+\left(\frac{v}{6}-1\right) \beta(t)\right\} \\
& \leq \beta(t)+\left\{\frac{1}{n_{0}}+\left(\frac{v}{6}-1\right) \frac{1}{v+1}\right\} \leq \beta(t) \quad \text { for } t \in(0,1)
\end{aligned}
$$

since $v /(\nu+1) \leq 1$ and $(v / 6-1) /(\nu+1)+1 / n_{0} \leq 0$. Finally (2.10) with $g(y)=$ $1 / y^{1 / \nu}$ (or (2.11) since if $0<x<y$ then $x^{1 / v}<y^{1 / v}$ ) holds. The existence of a solution $y$ to (2.26) follows from Theorem 2.1 (I) (or (II)). Note as well that $y(t) \geq \alpha(t)$ for $t \in[0,1]$ where $\alpha$ is given in (2.28).

\section{Initial value problems}

In this section we consider the initial boundary value problem

$$
\left\{\begin{array}{l}
y^{\prime}=q f(t, y), \quad 0<t<T(<\infty) \\
y(0)=0 .
\end{array}\right.
$$

Our results in this section differ from those in [4], that is, instead of assuming the existence of a lower solution to the singular problem (which is difficult to construct in practice) as in [4] we assume only the existence of a lower solution to the "approximating nonsingular problem". For our first result in this section we assume the following conditions are satisfied:

$$
\begin{gathered}
f:[0, T] \times(0, \infty) \rightarrow \mathbf{R} \text { is continuous } \\
q \in C(0, T], \quad q>0 \text { on }(0, T] \text { and } \int_{0}^{T} q(x) d x<\infty
\end{gathered}
$$

$$
\begin{gathered}
\left\{\begin{array}{l}
\exists n_{0} \in[1,2, \ldots\} \text { and associated with each } m \in N_{0}=\left\{n_{0}, n_{0}+1, \ldots\right\}, \\
\exists \alpha_{m} \in C[0, T] \cap C^{1}(0, T] \text { with } \\
q(t) f\left(t, \alpha_{m}(t)\right) \geq \alpha_{m}^{\prime}(t) \text { for } t \in(0, T) \text { and } 0<\alpha_{m}(0) \leq 1 / m
\end{array}\right. \\
\qquad\left\{\begin{array}{l}
\exists \alpha \in C[0, T], \alpha>0 \text { on }(0, T] \text { and } \alpha(t) \leq \alpha_{m}(t), \\
t \in[0, T] \text { for each } m \in N_{0}
\end{array}\right. \\
\left\{\begin{array}{l}
\exists \beta \in C[0, T] \cap C^{\prime}(0, T] \text { with } q(t) f(t, \beta(t)) \leq \beta^{\prime}(t) \\
\text { for } t \in(0, T) \text { and } \beta(0) \geq \beta_{0}>0
\end{array}\right.
\end{gathered}
$$

and

$$
\alpha_{m}(t) \leq \beta(t), \quad t \in[0, T] \text { for each } m \in N_{0}
$$


THEOREM 3.1. (I) Suppose (3.2)-(3.7) hold and in addition assume the following condition is satisfied:

$$
\left\{\begin{array}{l}
|f(t, y)| \leq g(y) \text { on }[0, T] \times\left(0, a_{0}\right] \text { with } g>0 \\
\text { continuous and nonincreasing on }(0, \infty)
\end{array}\right.
$$

here $a_{0}=\sup _{t \in[0, T]} \beta(t)$. Then (3.1) has a solution $y \in C[0, T] \cap C^{1}(0, T]$ with $y(t) \geq \alpha(t)$ for $t \in[0, T]$.

(II) Suppose (3.2)-(3.7) hold and in addition assume the following condition is satisfied:

$$
f(t, x)-f(t, y) \geq 0 \text { for } 0<x<y, \text { for each fixed } t \in(0, T) .
$$

Then (3.1) has a solution $y \in C[0, T] \cap C^{1}(0, T]$ with $y(t) \geq \alpha(t)$ for $t \in[0, T]$.

PROOF. Without loss of generality assume $\beta_{0} \geq 1 / n_{0}$. Fix $m \in N_{0}$ and consider

$$
\left\{\begin{array}{l}
y^{\prime}=q f_{m}^{\star}(t, y), \quad 0<t<T \\
y(0)=1 / m
\end{array}\right.
$$

where

$$
f_{m}^{\star}(t, y)= \begin{cases}f(t, \beta(t)), & y>\beta(t) \\ f(t, y), & \alpha_{m}(t) \leq y \leq \beta(t) \\ f\left(t, \alpha_{m}(t)\right), & y<\alpha_{m}(t) .\end{cases}
$$

It is immediate from Schauder's fixed point theorem (see [10]) that $(3.10)^{m}$ has a solution $y_{m} \in C[0, T]$. A standard argument (see [11, Chapter 3]; note $f_{m}^{*}$ : $[0,1] \times \mathbf{R} \rightarrow \mathbf{R}$ is continuous) guarantees that

$$
\alpha_{m}(t) \leq y_{m}(t) \leq \beta(t) \text { for } t \in[0, T] .
$$

As a result $y_{m}$ is a solution of

$$
\left\{\begin{array}{l}
y^{\prime}=q f(t, y), \quad 0<t<T \\
y(0)=1 / m
\end{array}\right.
$$

with

$$
\alpha(t) \leq \alpha_{m}(t) \leq y_{m}(t) \leq \beta(t) \text { for } t \in[0, T] .
$$

The proof is now broken into two cases.

Case (A). Suppose (3.8) holds.

We first show

$$
\left\{y_{m}\right\}_{m \in N_{0}} \text { is a bounded, equicontinuous family on }[0, T] .
$$


To see this notice (3.8) guarantees that $\left|y_{m}^{\prime}(t)\right| / g\left(y_{m}(t)\right) \leq q(t)$ for $t \in(0, T)$, and so $\pm v_{m}^{\prime}(t) \leq q(t)$ for $t \in(0, T)$; here

$$
v_{m}(t)=\int_{0}^{y_{m}(t)} \frac{d u}{g(u)}=G\left(y_{m}(t)\right)
$$

For $t, s \in[0, T]$ we have

$$
\left|v_{m}(t)-v_{m}(s)\right|=\left|\int_{s}^{t} v_{m}^{\prime}(\tau) d \tau\right| \leq\left|\int_{s}^{t} q(\tau) d \tau\right| .
$$

This together with the uniform continuity of $G^{-1}$ on $\left[0, G\left(a_{0}\right)\right]$ and

$$
\left|y_{m}(t)-y_{m}(s)\right|=\left|G^{-1}\left(G\left(y_{m}(t)\right)\right)-G^{-1}\left(G\left(y_{m}(s)\right)\right)\right|
$$

immediately guarantees (3.14). A standard argument [4, page 53] using the ArzelaAscoli theorem completes the proof.

Case (B). Suppose (3.9) holds.

We begin by showing

$$
y_{m+1}(t) \leq y_{m}(t) \text { for } t \in[0, T] \text { for each } m \in N_{0} .
$$

Suppose (3.15) is false. Then for some $m \in N_{0}$ there exists $\tau_{1}<\tau_{2}$ with $y_{m+1}\left(\tau_{1}\right)=$ $y_{m}\left(\tau_{1}\right), y_{m+1}\left(\tau_{2}\right)>y_{m}\left(\tau_{2}\right)$ and $y_{m+1}(t)>y_{m}(t)$ for $t \in\left(\tau_{1}, \tau_{2}\right)$. As a result from (3.9) we have

$$
0<y_{m+1}\left(\tau_{2}\right)-y_{m}\left(\tau_{2}\right)=\int_{\tau_{1}}^{\tau_{2}} q(s)\left[f\left(s, y_{m+1}(s)\right)-f\left(s, y_{m}(s)\right)\right] d s \leq 0,
$$

a contradiction. As a result (3.15) holds.

Essentially the same reasoning as in Theorem 2.1 guarantees that there exist subsequences of integers $N_{n_{0}} \supseteq N_{n_{0}+1} \supseteq \cdots \supseteq N_{k} \supseteq \cdots$ and functions $z_{k} \in C[T / k, T]$ with $y_{m}$ converging uniformly on $[T / k, T]$ to $z_{k}$ as $m \rightarrow \infty$ through $N_{k}$, and $z_{k+1}=z_{k}$ on $[T / k, T]$.

Define a function $y:[0, T] \rightarrow[0, \infty)$ by $y(x)=z_{k}(x)$ on $[T / k, T]$ and $y(0)=0$. Notice $y$ is well-defined and $\alpha(t) \leq y(t) \leq a_{0}$ for $t \in(0, T]$. Next fix $t \in(0, T)$ and let $k \in\left\{n_{0}, n_{0}+1, \ldots\right\}$ be such that $T / k<t<T$. Let $N_{k}^{*}=\left\{n \in N_{k}: n \geq k\right\}$. Now $y_{m}, m \in N_{k}^{*}$, satisfies

$$
y_{m}(t)=y_{m}(T)-\int_{t}^{T} q(s) f\left(s, y_{m}(s)\right) d s .
$$

Let $m \rightarrow \infty$ through $N_{k}^{*}$ to obtain $y(t)=y(T)-\int_{t}^{T} q(s) f(s, y(s)) d s$. We can do this argument for each $t \in(0, T)$. It remains to show $y$ is continuous at 0 . Let $\epsilon>0$ 
be given. Then there exists $n_{1} \in N_{0}$ with $y_{n_{1}}(0)<\epsilon / 2$, so there exists $\delta_{n_{1}}>0$ with $y_{n_{1}}(t)<\epsilon / 2$ for $t \in\left[0, \delta_{n_{1}}\right]$. From (3.15) for $m \geq n_{1}$ we have

$$
\alpha(t) \leq y_{m}(t) \leq y_{n_{1}}(t)<\epsilon / 2 \text { for } t \in\left[0, \delta_{n_{1}}\right]
$$

As a result $0 \leq \alpha(t) \leq y(t) \leq \epsilon / 2<\epsilon$ for $t \in\left(0, \delta_{n_{1}}\right]$, so $y$ is continuous at 0 .

In fact one can obtain a more general result motivated from Theorem 3.1 (II).

THEOREM 3.2. Suppose (3.2)-(3.5) hold and in addition assume the following conditions are satisfied:

$$
\left\{\begin{array}{c}
\text { for each } m \in N_{0}, \exists \beta_{m} \in C[0, T] \cap C^{1}(0, T] \text { with } \\
q(t) f\left(t, \beta_{m}(t)\right) \leq \beta_{m}^{\prime}(t) \text { for } t \in(0, T) \text { and } \beta_{m}(0) \geq 1 / m \\
\alpha_{m}(t) \leq \beta_{m}(t), \quad t \in[0, T] \text { for each } m \in N_{0}
\end{array}\right.
$$

and

$$
\left\{\begin{array}{l}
\text { for each } t \in[0, T] \text { we have that }\left\{\beta_{m}(t)\right\}_{m \in N_{0}} \text { is a } \\
\text { nonincreasing sequence and } \lim _{m \rightarrow \infty} \beta_{m}(0)=0 .
\end{array}\right.
$$

Then (3.1) has a solution $y \in C[0, T] \cap C^{l}(0, T]$ with $y(t) \geq \alpha(t)$ for $t \in[0, T]$.

PROOF. Fix $m \in N_{0}$. Proceed as in Theorem 3.1 with $\beta_{m}$ replacing $\beta$ in $f_{m}^{\star}$. The same reasoning as in Theorem 3.1 guarantees that there exists a solution $y_{m} \in C[0, T]$ to (3.12) with $\alpha(t) \leq \alpha_{m}(t) \leq y_{m}(t) \leq \beta_{m}(t)$ for $t \in[0, T]$. Also as in Theorem 3.1 there exists $y \in C(0, T]$ (as described in Theorem 3.1 (II)) with

$$
\alpha(t) \leq y(t) \leq a_{0}=\sup _{t \in[0, T]} \beta_{n_{0}}(t) \quad \text { for } t \in(0, T]
$$

with $y^{\prime}=q f(t, y)$ for $0<t<T$. It is easy to see (using (3.18)) that $y$ is continuous at 0 .

\section{References}

[1] A. Acrivos, M. J. Shah and E. E. Peterson, "Momentum and heat transfer in laminar boundary layer flows of non-Newtonian fluid past external surfaces", AIChE J. 6 (1960) 312-317.

[2] R. P. Agarwal and D. O'Regan, "Nonlinear superlinear singular and nonsingular second order boundary value problems", J. Diff. Eqns 143 (1998) 60-95.

[3] R. P. Agarwal and D. O'Regan, "Singular initial and boundary value problems with sign changing nonlinearities", IMA J. Appl. Math. 65 (2000) 173-198. 
[4] R. P. Agarwal and D. O'Regan, "A note on upper and lower solutions for singular initial value problems", Math. Methods Appl. Sci. 24 (2001) 49-57.

[5] R. P. Agarwal, D. O'Regan, V. Lakshmikantham and S. Leela, "An upper and lower solution theory for singular Emden-Fowler equations", Nonlinear Anal. Real World Appl. 3 (2002) 275-291.

[6] L. E. Bobisud, J. E. Calvert and W. D. Royalty, "Some existence results for singular boundary value problems", Diff. Integral Eqns 6 (1993) 553-571.

[7] P. W. Eloe and J. Henderson, "Singular nonlinear boundary value problems for higher order ordinary differential equations", Nonlinear Anal. 17 (1991) 1-10.

[8] L. H. Erbe and H. Wang, "On the existence of positive solutions of ordinary differential equations", Proc. Amer. Math. Soc. 120 (1994) 743-748.

[9] A. Nachman and A. Callegari, "A nonlinear singular boundary value problem in the theory of pseudoplastic fluids", SIAM J. Appl. Math. 38 (1980) 275-282.

[10] D. O'Regan, Theory of singular boundary value problems (World Scientific, Singapore, 1994).

[11] D. O'Regan, Existence theory for nonlinear ordinary differential equations (Kluwer Academic Publishers, Dordrecht, 1997). 